\title{
Synthesis of High-Quality Carbon Nanotube Arrays without the Assistance of Water
}

\author{
Yongfeng Luo, ${ }^{1,2}$ Xinjun Wang, ${ }^{2}$ Mengdong He, ${ }^{2} \mathrm{Xi} \mathrm{Li}^{2}$ and Hong Chen ${ }^{2}$ \\ ${ }^{1}$ Institute of Powder Metallurgy, Central South University, Hunan, Changsha 410083, China \\ ${ }^{2}$ Institute of Mathematics and Physics, Central South University of Forestry and Technology, Hunan, Changsha 410004, China
}

Correspondence should be addressed to Yongfeng Luo, lyfnku@163.com and Hong Chen, chenhongcs@126.com

Received 2 November 2011; Accepted 5 February 2012

Academic Editor: Arava Leela Mohana Reddy

Copyright (C) 2012 Yongfeng Luo et al. This is an open access article distributed under the Creative Commons Attribution License, which permits unrestricted use, distribution, and reproduction in any medium, provided the original work is properly cited.

\begin{abstract}
Long and high-quality carbon nanotube (CNT) arrays have been synthesized through a chemical vapor deposition process. The $\mathrm{Fe} / \mathrm{Al}_{2} \mathrm{O}_{3}$ on silicon was used as the catalyst, ethylene as the carbon source, and a gas mixture of $\mathrm{Ar}$ and $\mathrm{H}_{2}$ gases as the carrying gas. It is found for the first time that the high-quality and superlong carbon nanotube array can be improved by varying the content of hydrogen and carbon source.
\end{abstract}

\section{Introduction}

Carbon nanotubes (CNTs) have been extensively studied recently due to their unique structures and excellent mechanical, electrical, and chemical properties [1-14]. The special characteristics of carbon nanotubes arise from their atomic structures, number of walls, diameters, and lengths. For example, CNTs can be either metals or semiconductors depending on chirality and diameters $[15,16]$. The alignment, diameter, and number of walls of a CNT significantly affect its mechanical and electrical properties and thus can impact a wide range of applications such as probe microscopy tips $[17,18]$, field emission devices [19], solar cells $[7,20]$, electromechanical devices [21], and structural composites $[22,23]$. Recently, new technology based on the properties of very long aligned CNTs has been developing rapidly. The long nanotubes can be spun into fibers [20, 24-30] that are much stronger than all current structural materials. This will allow revolutionary advances in lightweight and high-strength applications. Intense research efforts have been undertaken to synthesize aligned long CNTs [31-38]; nevertheless, many limitations to the synthesis of very long aligned CNTs remain. Recently, the Iijima [38] group succeeded in growing $2.5 \mathrm{~mm}$ long aligned singlewalled carbon nanotubes of high purity using the waterassisted chemical vapor deposition (CVD) technique. For the synthesis of very long aligned CNTs by CVD technique, the catalyst activity and lifetime are very important factors. The coating of the catalyst particles by amorphous carbon during CVD reduces the catalyst activity and stops the growth of CNTs. The successful synthesis of very long CNTs can be achieved if the catalyst activity and lifetime can be enhanced [38]. The control of the diameter and number of walls of CNTs represents one of the most basic issues in developing nanotube growth methods. There are some reports that the size of the catalyst used in thermal catalytic CVD can define the diameter of as-grown nanotubes [3941]. This hypothesis has been supported by the observation that catalytic particles at the ends of CVD-grown CNTs have sizes commensurate with the nanotube diameters. The direct growth of long CNTs with a tunable number of walls by adjusting the catalyst thickness can be very promising due to its simplicity and the possibility of wide applications, which have not been yet reported. Accordingly, the developments of synthesis techniques that can control the length, diameter, alignment, and number of walls of the nanotubes have become the most important part of intense research. In the present study, we report the successful synthesis of $4 \mathrm{~mm}$-long aligned CNTs by improving the flow ratio of hydrogen in the catalytic thermal CVD technique without waterassistance. 


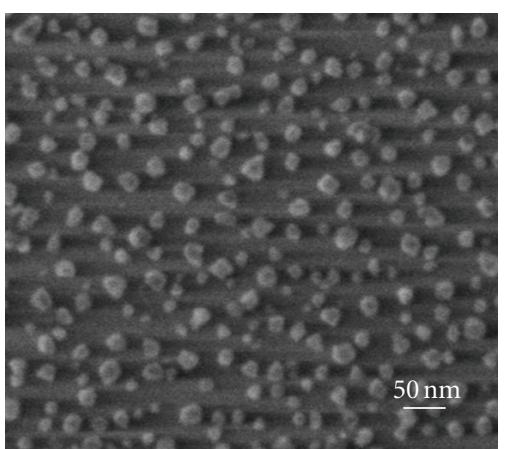

(a)

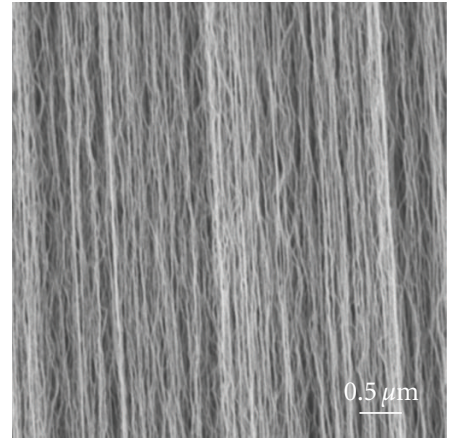

(b)

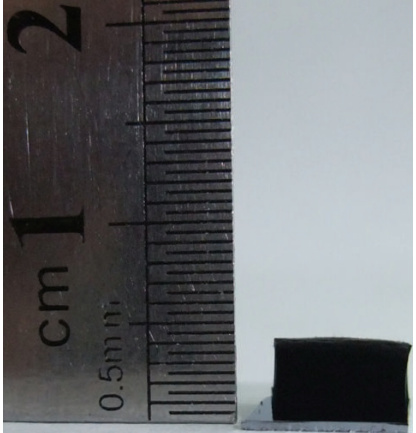

(c)

FIGURE 1: (a) SEM images of the catalytic iron nanoparticles after the heating treatment in simulating the CNT synthesis without introduction of the carbon source; (b) SEM image showing sidewall of well-aligned CNTs after the growth for 180 min; (c) optical image of $4.0 \mathrm{~mm}$ long CNT arrays after the growth for $180 \mathrm{~min}$.

\section{Experimental}

The long aligned CNTs were synthesized using a conventional CVD technique. High-quality and long CNTs arrays were synthesized in an atmospheric pressure quartz tube furnace having the inner diameter of $47 \mathrm{~mm}$. Ar with $30 \%$ $\mathrm{H}_{2}$ was used as a carrier gas, and pure ethylene served as the carbon source. CNTs growth was performed in quartz tube furnace. High-purity ethylene (99.999\%) was used as the source gas of carbon. Argon (99.999\%) was used as a carrier gas, and hydrogen (99.999\%) was introduced with $\mathrm{Ar}$ at 1 atm pressure.

Typical CVD growth was carried out at $750^{\circ} \mathrm{C}$. During heating of the reactor, a steady flow of $300 \mathrm{sccm}$ argon and $150 \mathrm{sccm} \mathrm{H}_{2}$ was maintained into the chamber. After the growth temperature was attained, the total gas flow rate throughout the deposition process was maintained at $500 \mathrm{sccm}$. The optimum CVD condition was determined by balancing the relative levels of ethylene and $\mathrm{H}_{2}$. This sharp optimum condition was achieved for the growth of long aligned CNTs array by studying the dependence of the height of CNTs on ethylene-to- $\mathrm{H}_{2}$ ratios, which has been reported earlier. In contrast to the report of Hata et al. [38] where the catalyst was active only for $30 \mathrm{~min}$, we achieved very long time (ca. $3 \mathrm{~h}$ ) catalyst activity by optimizing the CVD parameters very accurately, which resulted in the growth of aligned CNTs as long as $4 \mathrm{~mm}$. Si wafers $(5 \mathrm{~mm} \times 5 \mathrm{~mm})$ with $1 \mu \mathrm{m}$ thickness $\mathrm{SiO}_{2}$ layer were used as the substrate for the growth of long CNTs. The $\mathrm{Al}_{2} \mathrm{O}_{3}$ layer of thicknesses $(10 \mathrm{~nm}$ ) was used as a buffer layer between the $\mathrm{Si} / \mathrm{SiO}_{2}$ substrate and the catalyst. The $\mathrm{Al}_{2} \mathrm{O}_{3}$ layer was deposited on the substrate on which thicknesses $(1.0 \mathrm{~nm})$ of Fe catalyst layer were deposited to grow CNTs. Both the catalyst and buffer layers were deposited by an e-beam evaporation technique. The progress was normally for few seconds $(5 \mathrm{~s}$ for $1.0 \mathrm{~nm}$ thick Fe catalyst) to achieve catalyst thicknesses. The variation of Fe catalyst thickness was found to be most effective for controlling the number of walls of the aligned CNTs. The CNTs array with thicknesses up to $4.0 \mathrm{~mm}$ can be synthesized by improving the flow ratio of hydrogen to $30 \%$. Amount of hydrogen is much larger than the $6 \%$ of total flow gas reported previously.

The microstructures of the deposited nanotubes were studied by scanning electron microscopy (SEM, Hitachi FESEM S4800 operated at $1 \mathrm{kV}$ ) and transmission electron microscopy (TEM, JEOL JEM-2100F operated at $200 \mathrm{kV}$ ). TEM samples were prepared by drop-casting CNT/ethanol solutions onto copper grids in the open air. Raman measurements were performed on Renishaw in Via Reflex with excitation wavelength of $514.5 \mathrm{~nm}$ and laser power of $20 \mathrm{~mW}$ at room temperature.

\section{Results and Discussion}

Long CNTs array up to $4.0 \mathrm{~mm}$ was synthesized through a chemical vapor deposition process. The experimental results showed that the synthesis of aligned CNTs is very sensitive to the CVD parameters. The large amount of hydrogen during CVD plays a critical role in synthesizing long aligned CNTs. The hydrogen acts like a weak oxidizer and helps to remove the amorphous carbon deposited on the catalyst surface during CVD. The deposition of amorphous carbon layer on the catalyst surface reduces the activity and lifetime of the catalyst; as a result long continuous CNTs cannot be synthesized. By optimizing the CVD parameters and the hydrogen concentration, it is possible to grow long CNTs with high reproducibility.

It seems that upon heating to CNTs growth temperature the $\mathrm{Fe}$ nanoparticles formation occurred from the thin $\mathrm{Fe}$ layer. These particles were then trapped in the holes of the $\mathrm{Al}_{2} \mathrm{O}_{3}$ surface. The shape of the metal particles was also guided by the dip traps of the $\mathrm{Al}_{2} \mathrm{O}_{3}$ surface, and the appropriate shape of the nanoparticles is very important for growing high-quality CNTs. The surface morphology of the Fe-coated $\mathrm{Al}_{2} \mathrm{O}_{3}$ surface was also studied by scanning electron microscopy (SEM) (Figure 1(a)), and the Fe film breaks into uniform nanoparticles with an average size of about $14 \mathrm{~nm}$. SEM images of the aligned CNTs deposited for $30 \mathrm{~min}$ by using $\mathrm{Fe}$ catalyst layer $(1.0 \mathrm{~nm})$ are shown 


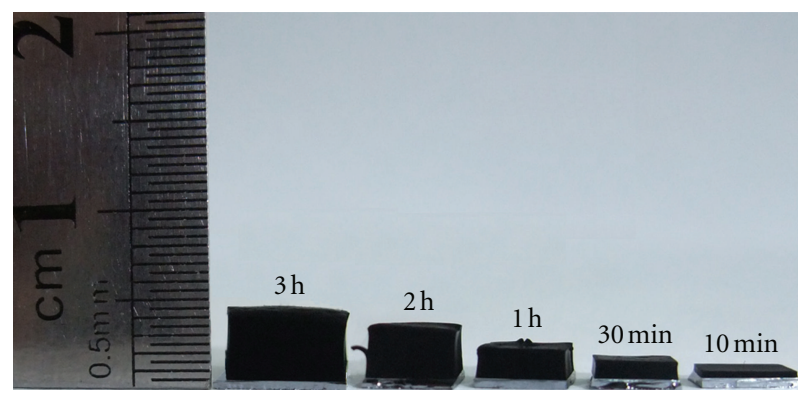

(a)

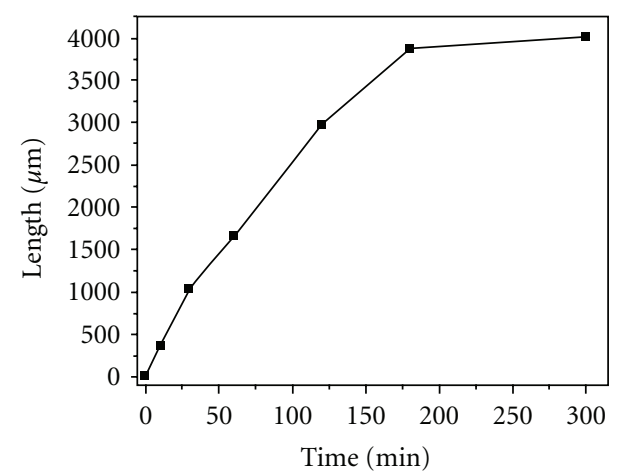

(b)

Figure 2: (a) and (b): growth length of CNT array with time at $750{ }^{\circ} \mathrm{C}$ and with ethylene and carrier-gas flow rates at $500 \mathrm{sccm}$.

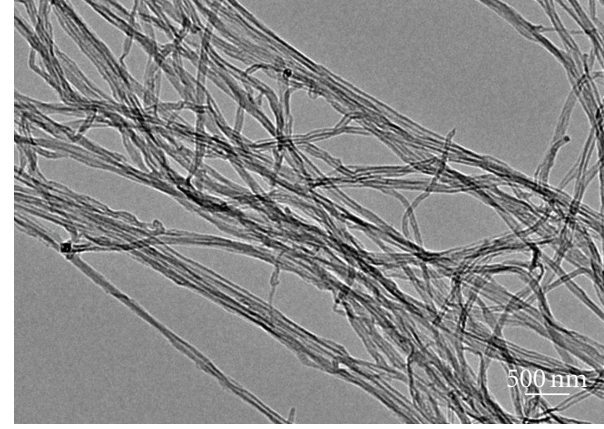

(a)

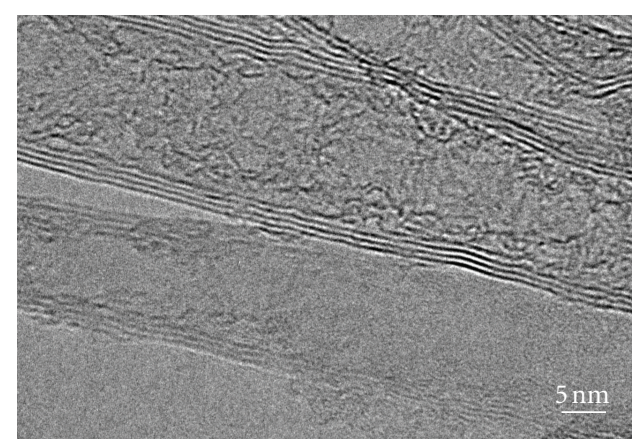

(b)

FIgURE 3: (a) Transmission electron microscopy (TEM) image of the typical CNTs. (b) High-resolution TEM image of the CNTs.

in Figure 1(b), which indicates high-quality aligned CNTs without any defect. Figure 1(c) shows that the height of aligned CNTs is up to $4 \mathrm{~mm}$.

Figure 2(a) compares the side views of the CNT arrays synthesized with different growth times of 10, 30, 60, 120, and $180 \mathrm{~min}$. The CNTs are highly aligned with each other in the above arrays. The plot of variation of growth rate with time for $5 \mathrm{~h}$ deposition is shown in Figure 2(b). The average growth rate is ca. $0.54 \mu \mathrm{m} / \mathrm{s}$ in the first 3 hours. In the case of the aligned CNTs grown from a $1.0 \mathrm{~nm}$ thick Fe layer, the growth rate of CNTs was found to not vary for long time. The plot indicates gradual slow decrease in the growth rate of CNTs in the first 3 hours. The growth rate is directly related to the catalyst activity during deposition. The balance between the rate of amorphous carbon deposition on the catalyst surface and removal of that amorphous carbon layer by hydrogen is the factor keeping the catalyst active for a long time. However, with increasing time the rate of amorphous carbon deposition increases compared to the rate of removal, which results in the decrease in the catalyst activity; the growth rate decreases followed by saturation, and finally the growth terminates due to the termination of catalyst activity. Figures $3(\mathrm{a})$ and 3(b), respectively, show the transmission electron microscopy (TEM) and high-resolution TEM images of individual CNTs grown for $180 \mathrm{~min}$. The outer diameters of CNTs are around
$12 \mathrm{~nm}$, and the inner diameters are about $9 \mathrm{~nm}$. The CNTs synthesized by this approach are all multi-walled with the interlamellar distance of $0.34 \mathrm{~nm}$.

The CNT arrays had been further investigated by Raman spectroscopy. The intensity ratios of $D$-band to $G$-band intensity ratio may reflect the structure integrity of the CNTs in the clean arrays. Figure 4(a) shows typical Raman spectra of the top surfaces in the CNTs arrays synthesized with the growth times of 10,60 , and $180 \mathrm{~min}$. The $D$-band and $G$-band are located at about $1337 \mathrm{~cm}^{-1}$ and $1571 \mathrm{~cm}^{-1}$, respectively. Figure 4 (b) carefully compares the $I_{D} / I_{G}$ values from the top of the CNTs arrays. It was found that the $I_{D} / I_{G}$ values remain almost unchanged with the increasing growth times for both cases. In other words, the synthesized CNTs show high structure integrity.

The growth mechanism of long CNT arrays had been previously investigated by several groups [28, 29]. For instance, Peng et al. [28] found that the catalyst played a critical role in the structure and size of the resulting CNTs during a chemical vapor deposition process. A tiny variation in the catalyst composition produced the so-called pearllike CNT which was very important to the spinnability of the CNT array. In this work, we also believe that the highperformance catalyst should be responsive for the highquality CNT array without the assistance of water. It was found that uniform nanoparticles could be produced upon 


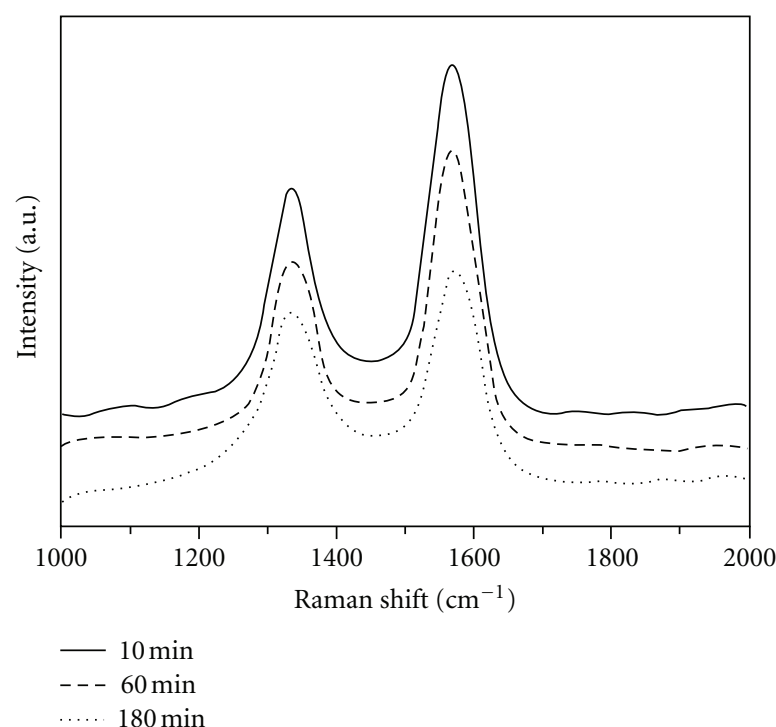

(a)

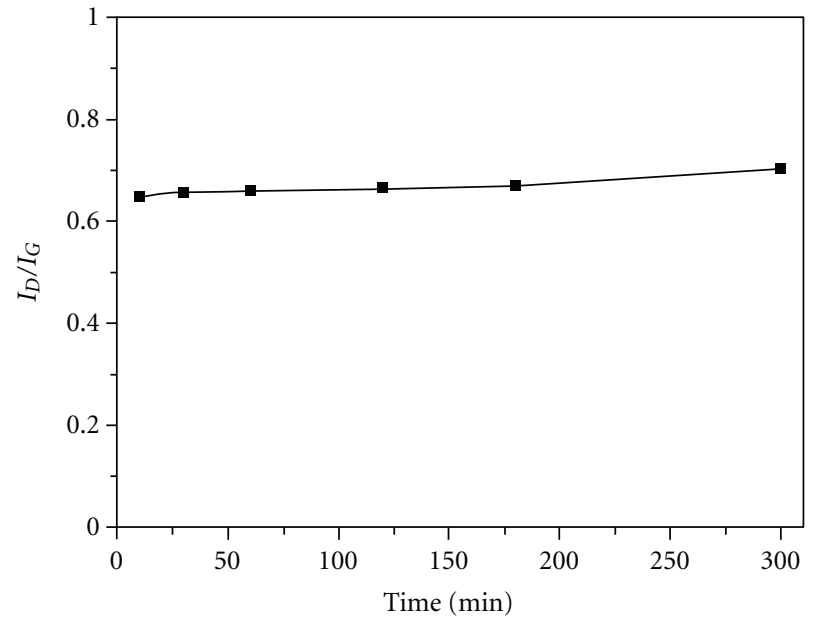

(b)

Figure 4: (a) Raman spectra of the CNT arrays grown for $10 \mathrm{~min}, 60 \mathrm{~min}$, and $180 \mathrm{~min}$. (b) Dependence of the $I_{D} / I_{G}$ on the growth time from the top of the CNT arrays. Here $I_{D}$ and $I_{G}$ correspond to the peak intensities of D-band and G-band, respectively.

heating treatment of the catalyst in mimicking the synthetic condition. More efforts are underway.

\section{Conclusions}

In summary, we have developed a catalyst structure consisting of a thin Fe film on a dense $\mathrm{Al}_{2} \mathrm{O}_{3}$ buffer layer that was deposited on a $\mathrm{Si}$ substrate by e-beam evaporation. This catalyst lifetime was prolonged (>120 min), resulting in growth of CNT arrays that are $4 \mathrm{~mm}$ long. These CNT lengths are much longer than those reported previously. Increasing fraction of hydrogen was found to reduce the formation of amorphous carbon and to extend the catalyst life. Our synthesis of long CNT arrays requires a very low fraction of ethylene and large fraction of hydrogen.

\section{Acknowledgments}

The authors thank L. Li, X. Sun, and L. Qiu for suggestive discussions. This work was supported by the National Natural Science Foundation of China (Grant no. 11174372).

\section{References}

[1] L. Zhu, D. W. Hess, and C. P. Wong, "Monitoring carbon nanotube growth by formation of nanotube stacks and investigation of the diffusion-controlled kinetics," Journal of Physical Chemistry B, vol. 110, no. 11, pp. 5445-5449, 2006.

[2] H. Peng, Y. Zhu, D. E. Peterson, and Y. Lu, "Nanolayered carbon/silica superstructures via organosilane assembly," Advanced Materials, vol. 20, no. 6, pp. 1199-1204, 2008.

[3] H. Peng, D. Chen, J. Y. Huang et al., "Strong and ductile colossal carbon tubes with walls of rectangular macropores," Physical Review Letters, vol. 101, no. 14, Article ID 145501, 2008.
[4] H. Peng and X. Sun, "Macroporous carbon nanotube arrays with tunable pore sizes and their template applications," Chemical Communications, no. 9, pp. 1058-1060, 2009.

[5] H. Peng and X. Sun, "Highly aligned carbon nanotube/ polymer composites with much improved electrical conductivities," Chemical Physics Letters, vol. 471, no. 1-3, pp. 103105, 2009.

[6] L. Li, Z. Yang, H. Gao et al., "Vertically aligned and penetrated carbon nanotube/polymer composite film and promising electronic applications," Advanced Materials, vol. 23, no. 32, pp. 3730-3735, 2011.

[7] S. Huang, L. Li, Z. Yang et al., "A new and general fabrication of aligned carbon nanotube/polymer film for electrode application," Advanced Materials, vol. 23, no. 40, pp. 4707-4710, 2011.

[8] H. Peng, X. Sun, F. Cai et al., "Electrochromatic carbon nanotube/polydiacetylene nanocomposite fibres," Nature Nanotechnology, vol. 4, no. 11, pp. 738-741, 2009.

[9] H. Peng, M. Jain, D. E. Peterson, Y. Zhu, and Q. Jia, "Composite carbon nanotube/silica fibers with improved mechanical strengths and electrical conductivities," Small, vol. 4, no. 11, pp. 1964-1967, 2008.

[10] X. Sun, T. Chen, S. Huang, L. Li, and H. Peng, "Chromatic polydiacetylene with novel sensitivity," Chemical Society Reviews, vol. 39, no. 11, pp. 4244-4257, 2010.

[11] H. Peng, "Aligned carbon nanotube/polymer composite films with robust flexibility, high transparency, and excellent conductivity," Journal of the American Chemical Society, vol. 130, no. 1, pp. 42-43, 2008.

[12] T. Chen, S. Wang, Z. Yang et al., "Flexible, light-weight, ultrastrong, and semiconductive carbon nanotube fiber for highly efficient novel solar cell," Angewandte Chemie International Edition, vol. 50, no. 8, pp. 1815-1819, 2011.

[13] M. Zhang, S. Fang, A. A. Zakhidov et al., "Materials science: strong, transparent, multifunctional, carbon nanotube sheets," Science, vol. 309, no. 5738, pp. 1215-1219, 2005. 
[14] R. H. Baughman, A. A. Zakhidov, and W. A. De Heer, "Carbon nanotubes - the route toward applications," Science, vol. 297, no. 5582, pp. 787-792, 2002.

[15] T. W. Odom, J. L. Huang, P. Kim, and C. M. Lieber, "Atomic structure and electronic properties of single-walled carbon nanotubes," Nature, vol. 391, no. 6662, pp. 62-64, 1998.

[16] J. W. G. Wildöer, L. C. Venema, A. G. Rinzler, R. E. Smalley, and C. Dekker, "Electronic structure of atomically resolved carbon nanotubes," Nature, vol. 391, no. 6662, pp. 59-62, 1998.

[17] H. Nishijima, S. Kamo, S. Akita et al., "Carbon-nanotube tips for scanning probe microscopy: preparation by a controlled process and observation of deoxyribonucleic acid," Applied Physics Letters, vol. 74, no. 26, pp. 4061-4063, 1999.

[18] S. Akita, Y. Nakayama, S. Mizooka et al., "Nanotweezers consisting of carbon nanotubes operating in an atomic force microscope," Applied Physics Letters, vol. 79, no. 11, pp. 16911693, 2001.

[19] H. Tanaka, S. Akita, L. Pan, and Y. Nakayama, "Daisylike field-emission images from standalone open-ended carbon nanotube," Japanese Journal of Applied Physics, Part 2, vol. 43, no. 2, pp. L197-L199, 2004.

[20] T. Chen, Z. Cai, Z. Yang et al., "Nitrogen-doped carbon nanotube composite fiber with a core-sheath structure for novel electrode," Advanced Materials, vol. 23, no. 40, pp. 46204625, 2011.

[21] O. Suekane, A. Nagataki, and Y. Nakayama, "Current-induced curing of defective carbon nanotubes," Applied Physics Letters, vol. 89, no. 18, Article ID 183110, 2006.

[22] J. Suhr, N. Koratkar, P. Keblinski, and P. Ajayan, "Viscoelasticity in carbon nanotube composites," Nature Materials, vol. 4, no. 2, pp. 134-137, 2005.

[23] B. J. Hinds, N. Chopra, T. Rantell, R. Andrews, V. Gavalas, and L. G. Bachas, "Aligned multiwalled carbon nanotube membranes," Science, vol. 303, no. 5654, pp. 62-65, 2004.

[24] Y. L. Li, I. A. Kinloch, and A. H. Windle, "Direct spinning of carbon nanotube fibers from chemical vapor deposition synthesis," Science, vol. 304, no. 5668, pp. 276-278, 2004.

[25] X. Zhang, K. Jiang, C. Feng et al., "Spinning and processing continuous yarns from 4-inch wafer scale super-aligned carbon nanotube arrays," Advanced Materials, vol. 18, no. 12, pp. 1505-1510, 2006.

[26] Z. Yang, X. Sun, X. Chen et al., "Structure and property dependence of carbon nanotube fiber on heating treatment," Journal of Materials Chemistry, vol. 21, no. 36, pp. 1377213775, 2011.

[27] M. Zhang, K. R. Atkinson, and R. H. Baughman, "Multifunctional carbon nanotube yarns by downsizing an ancient technology," Science, vol. 306, no. 5700, pp. 1358-1361, 2004.

[28] H. Peng, M. Jain, Q. Li, D. E. Peterson, Y. Zhu, and Q. Jia, "Vertically aligned pearl-like carbon nanotube arrays for fiber spinning," Journal of the American Chemical Society, vol. 130, no. 4, pp. 1130-1131, 2008.

[29] Q. Li, Y. Li, X. Zhang et al., "Structure-dependent electrical properties of carbon nanotube fibers," Advanced Materials, vol. 19, no. 20, pp. 3358-3363, 2007.

[30] K. Jiang, Q. Li, and S. Fan, "Spinning continuous carbon nanotube yarns," Nature, vol. 419, no. 6909, p. 801, 2002.

[31] H. M. Christen, A. A. Puretzky, H. Cui et al., "Rapid growth of long, vertically aligned carbon nanotubes through efficient catalyst optimization using metal film gradients," Nano Letters, vol. 4, no. 10, pp. 1939-1942, 2004.
[32] W. Z. Li, S. S. Xie, L. X. Qian et al., "Large-scale synthesis of aligned carbon nanotubes," Science, vol. 274, no. 5293, pp. 1701-1703, 1996.

[33] G. Y. Xiong, D. Z. Wang, and Z. F. Ren, "Aligned millimeterlong carbon nanotube arrays grown on single crystal magnesia," Carbon, vol. 44, no. 5, pp. 969-973, 2006.

[34] A. J. Hart and A. H. Slocum, "Rapid growth and flowmediated nucleation of millimeter-scale aligned carbon nanotube structures from a thin-film catalyst," Journal of Physical Chemistry B, vol. 110, no. 16, pp. 8250-8257, 2006.

[35] J. Liu, L. Yuan, X. Yang, A. Elbert, and A. T. Harris, "Synthesis of vertically aligned carbon nanotube arrays on polyhedral $\mathrm{Fe} / \mathrm{Al}_{2} \mathrm{O}_{3}$ catalysts," Chemical Communication, vol. 47, no. 22, pp. 6434-6436, 2011.

[36] C. Kocabas, M. Shim, and J. A. Rogers, "Spatially selective guided growth of high-coverage arrays and random networks of single-walled carbon nanotubes and their integration into electronic devices," Journal of the American Chemical Society, vol. 128, no. 14, pp. 4540-4541, 2006.

[37] X. Chen, L. Li, X. Sun, H. G. Kia, and H. Peng, "A novel synthesis of graphene nanoscrolls with tunable dimension at a large scale," Nanotechnology, vol. 23, no. 5, p. 55603, 2012.

[38] K. Hata, D. N. Futaba, K. Mizuno, T. Namai, M. Yumura, and S. Iijima, "Water-assisted highly efficient synthesis of impurity-free single-walled carbon nanotubes," Science, vol. 306, no. 5700, pp. 1362-1364, 2004.

[39] W. Guo, C. Liu, X. Sun, Z. Yang, H.G. Kia, and H. Peng, "Aligned carbon nanotube/polymer composite fibers with improved mechanical strength and electrical conductivity," Journal of Materials Chemistry, vol. 22, no. 3, pp. 903-908, 2012.

[40] Y. Y. Wei, G. Eres, V. I. Merkulov, and D. H. Lowndes, "Effect of catalyst film thickness on carbon nanotube growth by selective area chemical vapor deposition," Applied Physics Letters, vol. 78, no. 10, pp. 1394-1396, 2001.

[41] H. Dai, A. G. Rinzler, P. Nikolaev, A. Thess, D. T. Colbert, and R. E. Smalley, "Single-wall nanotubes produced by metalcatalyzed disproportionation of carbon monoxide," Chemical Physics Letters, vol. 260, no. 3-4, pp. 471-475, 1996. 

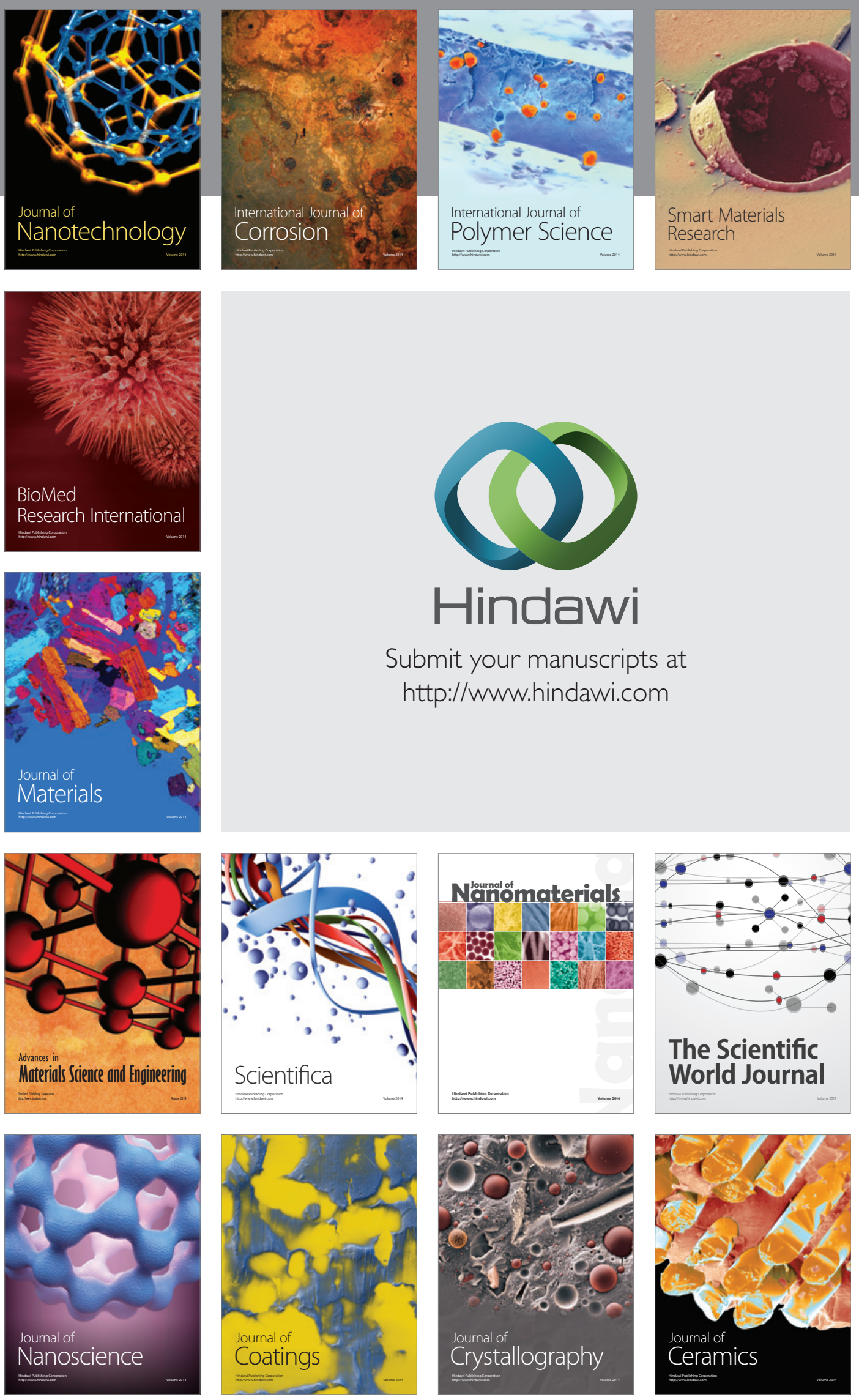

The Scientific World Journal

Submit your manuscripts at

http://www.hindawi.com

\section{World Journal}

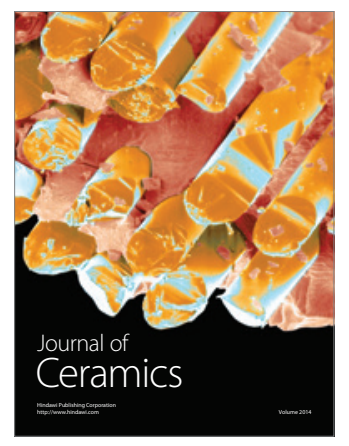

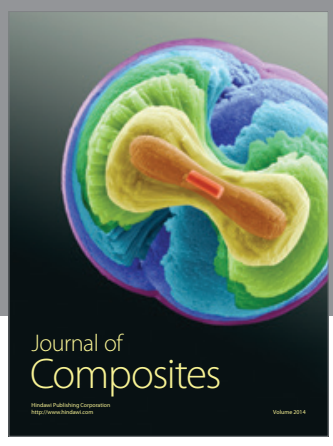
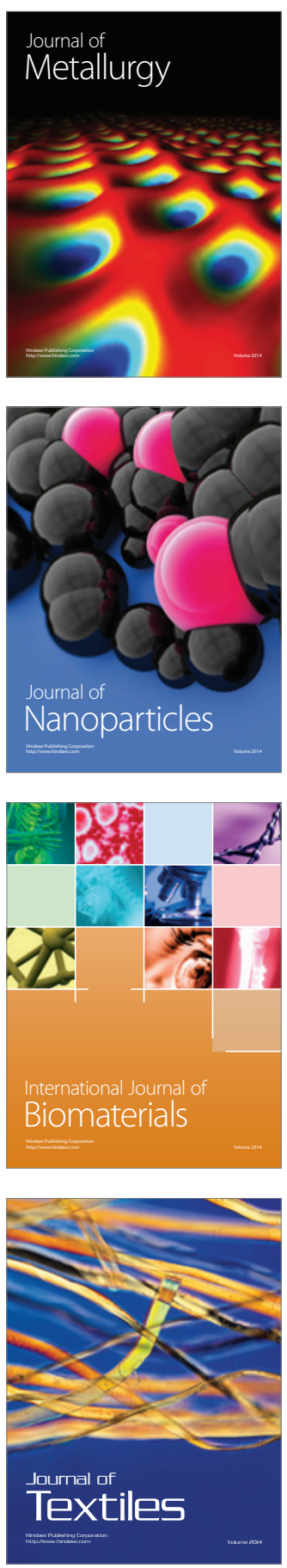